\title{
Neurorrestauración tras la isquemia cerebral: papel de los neurotransmisores en la neurogénesis postisquémica
}

\author{
Eduardo Sánchez-Mendoza, Víctor Bellver-Landete, María Pilar González, José Joaquín Merino, \\ Ricardo Martínez-Murillo, María Jesús Oset-Gasque
}

Introducción. La isquemia cerebral y la reperfusión producen alteraciones en el microambiente del parénquima, que incluyen la depleción de adenosín trifosfato, alteración de la homeostasis iónica, inflamación, liberación de múltiples citocinas y factores de crecimiento, y liberación anormal de neurotransmisores. Como consecuencia, se induce la proliferación y migración de las células precursoras neurales hacia las regiones del periinfarto.

Desarrollo. El éxito de los nuevos tratamientos neurorrestauradores para el cerebro dañado implica la necesidad de conocer con mayor precisión los mecanismos que regulan la neurogénesis de adulto, tanto en condiciones fisiológicas como patológicas. Evidencias recientes demuestran que muchos neurotransmisores, en especial el glutamato, afectan la zona subventricular, formando parte, por lo tanto, de la compleja red de señales que influencian la producción de nuevas neuronas.

Conclusiones. Los neurotransmisores proporcionan una conexión entre la actividad cerebral y la neurogénesis de la zona subventricular. Por ello, un conocimiento más profundo de la participación de los sistemas de neurotransmisores, como el glutamato y sus transportadores vesiculares y de membrana, en la neurogénesis del adulto puede proporcionar una herramienta valiosa que se podría utilizar como terapia neurorreparadora en esta patología.

Palabras clave. Células progenitoras neurales. Glutamato. Isquemia cerebral. Neurogénesis. Neurorreparación. Neurotransmisores.

\section{Introducción}

La única terapia vigente para el ictus está limitada al tratamiento con factor activador del plasminógeno tisular recombinante (rt-PA), que, aunque mejora el pronóstico funcional, sólo se emplea en menos de un 3\% de pacientes [1]. La neuroprotección, como estrategia para el tratamiento del ictus, eficaz en modelos experimentales, ha fracasado en estudios clínicos en fase III. La recuperación funcional de los pacientes con ictus se produce en una escala temporal superior a los seis meses, sólo explicable mediante mecanismos de neurorreparación mediados por fenómenos de plasticidad neuronal [2]. Tras un ictus, el pronóstico a los seis meses dependerá del tamaño y topografía de la lesión, así como del grado y eficacia de la plasticidad neuronal del individuo, última responsable de su recuperación funcional [3].

En el proceso de neurorreparación participan mecanismos moleculares y celulares de neurogénesis y sinaptogénesis, angiogénesis y gliogénesis, que dotan de funcionalidad a las neuronas de nueva formación [4], los cuales se disparan como consecuencia de las graves alteraciones homeostáticas inducidas por la falta de oxígeno y nutrientes y la liberación de factores tróficos en el período posterior de reperfusión.

Esta revisión se centra en los nuevos aspectos conocidos sobre la regulación de la neurogénesis del adulto y destaca el papel de los neurotransmisores como estrategia terapéutica neurorrestauradora en la enfermedad cerebrovascular.

\section{Neurogénesis en la zona subventricular del cerebro adulto y su regulación en la corriente migratoria rostral}

\section{Tipos celulares y marcadores de neurogénesis}

En el cerebro de mamíferos actualmente se reconocen dos zonas neurogénicas: la zona subgranular (ZSG) del giro dentado del hipocampo y la zona subventricular (ZSV) que cubre los ventrículos laterales (VL) [5]. La zona ventricular produce células madre neurales que forman la corteza durante el desarrollo embrionario. Una vez concluido el desarrollo, la zona ventricular se convierte en ZSV, que se separa del ventrículo por la capa de células ependimales
Departamento de Bioquímica y Biología Molecular II; Facultad de Farmacia; Universidad Complutense de Madrid (E. Sánchez-Mendoza, V. Bellver-Landete, M.P. González, J.J. Merino, M.J. Oset-Gasque). Departamento de Neurobiología Celular, Molecular y del Desarrollo; Instituto Cajal; Consejo Superior de Investigaciones Científicas ( $R$. Martínez-Murillo). Madrid, España.

Correspondencia:

Dra. María Jesús Oset Gasque. Departamento de Bioquímica y Biología Molecular II. Facultad de Farmacia. Universidad Complutense de Madrid. PI. Ramón y Cajal, s/n. Ciudad Universitaria. E-28040 Madrid.

Fax:

+34913941779.

E-mail:

mjoset@farm.ucm.es

Financiación:

MICINN (SAF2009-11219 y SAF2010-20337), ISCIII (Retic RENEVAS, RD06/0026/012) y UCM-Santander (GR35/10-B).

Aceptado tras revisión externa: 19.09.12.

Cómo citar este artículo: Sánchez-Mendoza E, BellverLandete V, González MP, Merino JJ, Martínez-Murillo R, Oset-Gasque MJ. Neurorrestauración tras la isquemia cerebral: papel de los neurotransmisores en la neurogénesis postisquémica. Rev Neurol 2012 55: 533-42. 
Figura 1. Comparación de la organización de la zona subventricular (ZSV) humana frente a la del ratón o rata: a) En humanos, la ZSV está conformada por cuatro capas diferenciadas: I, células ependimales; II, capa hipocelular; III, cinturón de astrocitos; y IV, zona de transición; b) En roedores, una monocapa de células ependimales separa el ventrículo del cerebro. Bajo ésta, los astrocitos forman el tubo glial por el que los neuroblastos migran en la corriente migratoria rostral. Adaptada de [6] (con permiso).
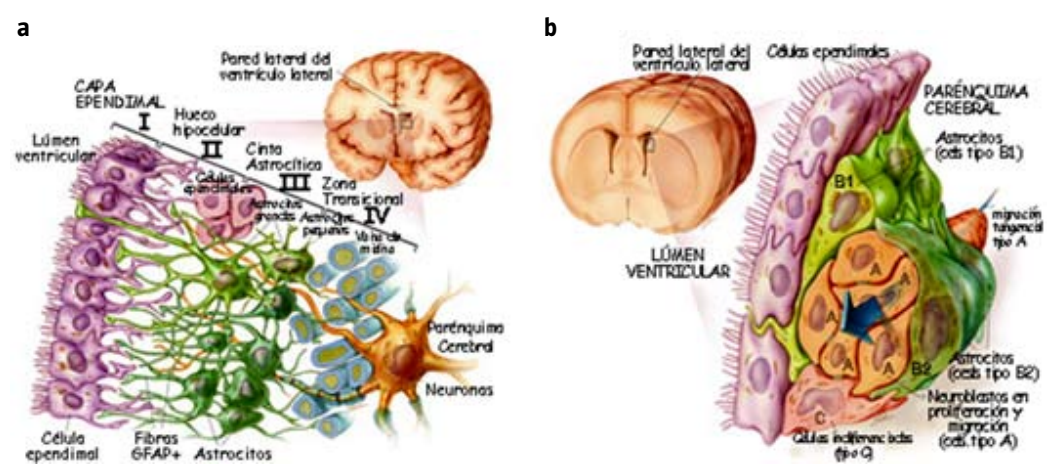

(Fig. 1) [5,6]. Por su relación con la isquemia, se hará referencia principalmente a la ZSV.

En humanos, la ZSV está formada por cuatro capas perfectamente organizadas (Fig. 1). Entre éstas, son de particular interés las capas II y III. La capa II no presenta cuerpos celulares, sino sitios de interconexión entre procesos de las células ependimales y de los astrocitos de la capa III, y sería un sitio donde la proliferación estaría regulada por células de la capa I y III en etapas tempranas de desarrollo, lo que constituiría un pasillo para la migración de células precursoras neurales (CPN). Por otro lado, se piensa que algunos de los astrocitos de la capa III podrían retener actividad proliferativa [6].

Existen tres tipos de células que participan en la neurogénesis en la ZSV (Fig. 2) [7,8]: las células tipo $B$ son células de tipo astrocitario que expresan proteína acídica gliofibrilar (GFAP), nestina y vimentina. Durante el proceso de división, algunas células B pasan a ser células de alta tasa de proliferación llamadas células $C$, y se caracterizan por la expresión de nestina. A partir de las células tipo $C$, se diferencian las células tipo A, que son los neuroblastos, que irán migrando a lo largo de la corriente migratoria rostral. Las células A se caracterizan por la expresión de $\beta$-3-tubulina (B3T), PSA-NCAM (forma polisialilada de la molécula de adhesión celular neuronal), doblecortina (DCX) y el factor de transcripción Dlx-2. Una vez que los neuroblastos alcanzan el bulbo olfatorio, se diferencian a interneuronas $[6,9]$.

El proceso de neurogénesis puede estudiarse in vitro mediante el cultivo de neuroesferas, estructu- ras de origen clonal, formadas in vitro, procedentes de las células madre neurales. Cada neuroesfera representa la progenie de una única célula madre que ha proliferado. De esta forma, la población de células que forman una neuroesfera es capaz de generar las tres líneas celulares principales presentes en el cerebro: neuronas, astrocitos y oligodendrocitos. En la figura 3 se aprecian imágenes de neuroesferas a tres días de diferenciación, tiempo en el que se incrementa la expresión de ARN mensajero de DCX y GFAP, mientras que disminuye la expresión de nestina [10].

\section{Regulación por factores de crecimiento y señales extracelulares}

Diversos estímulos, en especial los factores de crecimiento, influyen en este proceso, particularmente, el factor neurotrófico derivado del cerebro (BDNF), que regula la velocidad de migración de los neuroblastos hacia el bulbo olfatorio [11]. Sin embargo, existen muchos otros factores -factor de crecimiento de fibroblastos-2, factor de crecimiento epidérmico, factor de crecimiento transformante- $\beta$ (TGF $\beta)$, factor de crecimiento del endotelio vascular (VEGF), factor neurotrófico ciliar- que pueden modular diversos aspectos de la neurogénesis, aunque parecen estar implicados principalmente en la proliferación más que en la diferenciación [12]. Curiosamente, la liberación de BDNF desde astrocitos puede ser modulada por neurotransmisores como el glutamato, lo cual sugiere que debe explorarse la interacción de este neurotransmisor con los factores neurotróficos para entender mejor el proceso de neurogénesis en condiciones normales y patológicas [13].

Recientemente, Kaneko et al [14] han descrito la participación de la glucoproteína Slit1 y sus receptores ROBO en la migración a lo largo de la corriente migratoria rostral, mostrando que estas moléculas son esenciales en la organización del tubo glial por el que se desplazan los neuroblastos.

\section{Regulación por factores transcripcionales y receptores intracelulares}

Evidencias actuales ponen de manifiesto que la neurogénesis posnatal, tanto en la ZSV como en la ZSG, se regula por factores transcripcionales, como las neurogeninas, micro-ARN, DISC1 (gen de susceptibilidad a la esquizofrenia 1$)$ y CREB (cAMP response element binding protein), que están implicados en la proliferación, migración, supervivencia, diferenciación e integración sináptica de las CPN [15]. 
También se ha observado un papel muy relevante en la neurogénesis de distintos receptores nucleares (ER $\beta$, RAR $\alpha \beta \gamma$, PPAR $\gamma$, NURR1, TLX, SOX2, etc.), que actúan sobre distintos aspectos de la diferenciación y proliferación neuronal [16], siendo destacable la función del receptor nuclear huérfano TLX (NR2E1) sobre la capacidad de autorrenovación y proliferación de las $\mathrm{CPN}$ adultas, a través de un mecanismo de señalización que implica el reclutamiento de las histonadeacetilasas 3 y 5 (HDAC3 y HDAC5), y la represión transcripcional del inhibidor de Cdk's p21 y del gen supresor de tumores PTEN [17], junto con la activación de la ruta de Wnt/ $\beta$ catenina [18]. Por su parte, SOX2 activa la expresión de Hes5, que, en conjunto con la vía de Notch, induce la expresión de genes que retienen la diferenciación de las CPN, manteniéndolas en un estado proliferativo [19].

\section{Regulación por neurotransmisores}

Durante la última década se ha empezado a poner de manifiesto la importancia de neurotransmisores como el ácido $\gamma$-aminobutírico (GABA) y el glutamato, la serotonina, la dopamina o la acetilcolina en la regulación de la neurogénesis [20-24].

Las CPN expresan diferentes tipos de receptores de glutamato y GABA, dependiendo del estadio de desarrollo en que se encuentren, detectándose la presencia de tránscritos para diferentes subunidades de los receptores ionotrópicos $\mathrm{GABA}_{\mathrm{A}} \mathrm{y}$ $\mathrm{GABA}_{\mathrm{C}}$, y el metabotrópico $\mathrm{GABA}_{\mathrm{B}}$, así como para distintas subunidades de los receptores de NMDA, AMPA, kainato y para receptores metabotrópicos de los grupos I, II y III, en neuroesferas derivadas de la corteza [25].

En la ZSV, el GABA se sintetiza por las CPN y se libera al medio en respuesta a incrementos de $\mathrm{Ca}^{2+}$ que pueden producirse durante la migración o como respuesta a señales ambientales [26]. Puede funcionar como un regulador negativo de la proliferación y de la velocidad de migración de neuroblastos, y facilitar su formación de dendritas al incorporarse al bulbo olfatorio [27,28]. Por su parte, en la corriente migratoria rostral, el glutamato se libera, mediante un mecanismo dependiente de $\mathrm{Ca}^{2+}$, por el transportador vesicular VGLUT1 desde los astrocitos que rodean la corriente de neuroblastos [29]. La respuesta de las CPN y neuroblastos al glutamato depende del tipo de receptor activado y puede influir en múltiples aspectos de la neurogénesis. Así, la inhibición del receptor mGluR5 mediante $\alpha$-metil-4-carboxifenilglicina (MCPG) aumenta el número de neuroblastos que mueren por
Figura 2. Esquema del proceso de migración de células precursoras neurales desde la zona subventricular (SVZ) hacia el bulbo olfatorio (OB) a lo largo de la corriente migratoria rostral (RMS). Se muestran los tipos celulares que se pueden observar a lo largo del proceso y los marcadores moleculares específicos que se pueden utilizar para su inmunodetección. Las células B pueden ser astrocitos o células precursoras que se dividen generando nuevas células de división transitoria (células $C$ ), que, a su vez, se diferencian a neuroblastos (células $A$ ) y posteriormente a neuronas a medida que se desplazan por la corriente migratoria rostral. Modificada de [9] (con permiso).
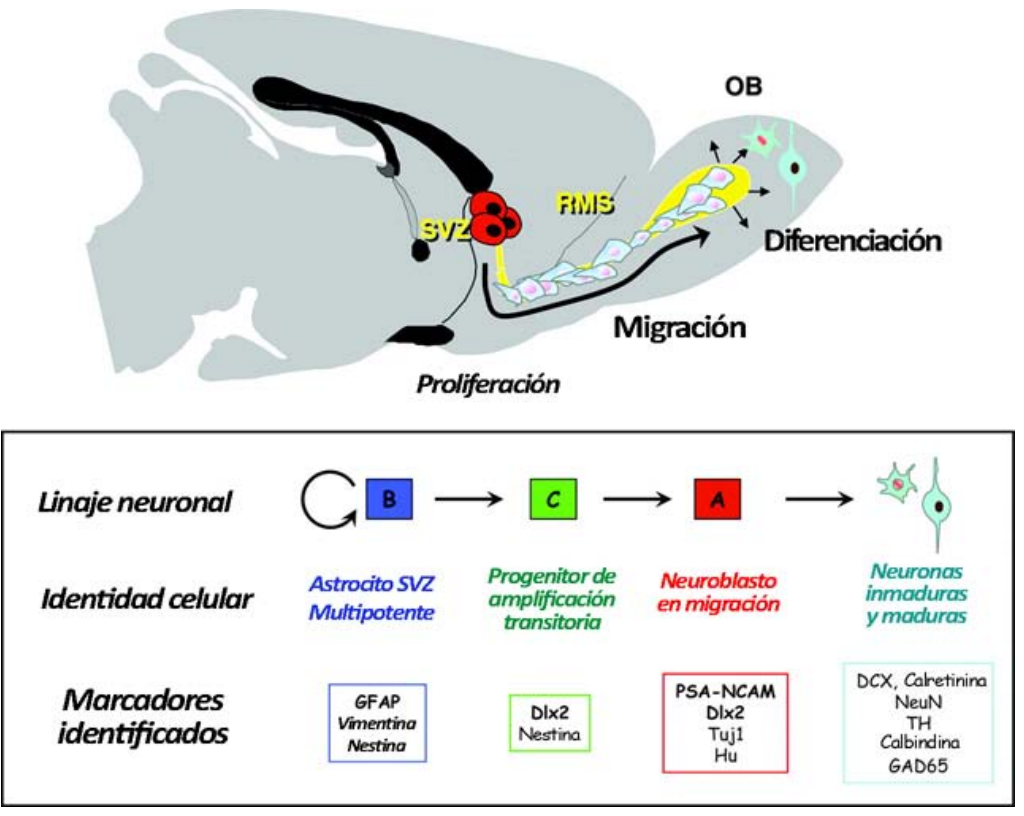

apoptosis, mientras que el inhibidor del receptor mGluR1, CPCCOEt, aumenta el número de neuroblastos viables [30]. Antagonistas del receptor de kainato GluR5 aceleran la migración de neuroblastos, pero la inhibición del receptor mGluR5 no altera este proceso [26]. Sin embargo, la activación del receptor mGluR5, tanto in vitro como in vivo, aumenta la proliferación de las CPN [31]. También se ha visto que el bloqueo selectivo del receptor NMDA reduce la supervivencia de neuroblastos en la corriente migratoria rostral [22]. En conjunto, estas observaciones indican no sólo que el glutamato participa activamente en la neurogénesis, sino que su efecto dependerá del balance de señales intracelulares activadas por los diferentes receptores.

Si bien la influencia del glutamato y el GABA es evidente, es importante considerar la posibilidad de que otros neurotransmisores también afecten el proceso de neurogénesis adulta tanto en animales sanos como en situaciones patológicas, destacando la influencia de la dopamina y la serotonina en las diferentes etapas de la neurogénesis. De hecho, las 
Figura 3. Aspecto de las neuroesferas de la zona subventricular tras tres días en cultivo primario: a) Imagen de neuroesferas en contrate de fases. Se aprecian células invadiendo el espacio intermedio entre ellas; b) Detalle de los neuroblastos (flechas) y células de aspecto glial (cabezas de flecha) obtenidos por microscopía de contraste de fases; c) Comparación de un neuroblasto y una neurona inmadura, ambas expresando doblecortina (DCX), marcadas mediante inmunocitoquímica con DAB; d): Evolución temporal de la expresión de los marcadores neurogénicos nestina, DCX y GFAP en neuroesferas en cultivo a uno, tres y siete días de diferenciación.
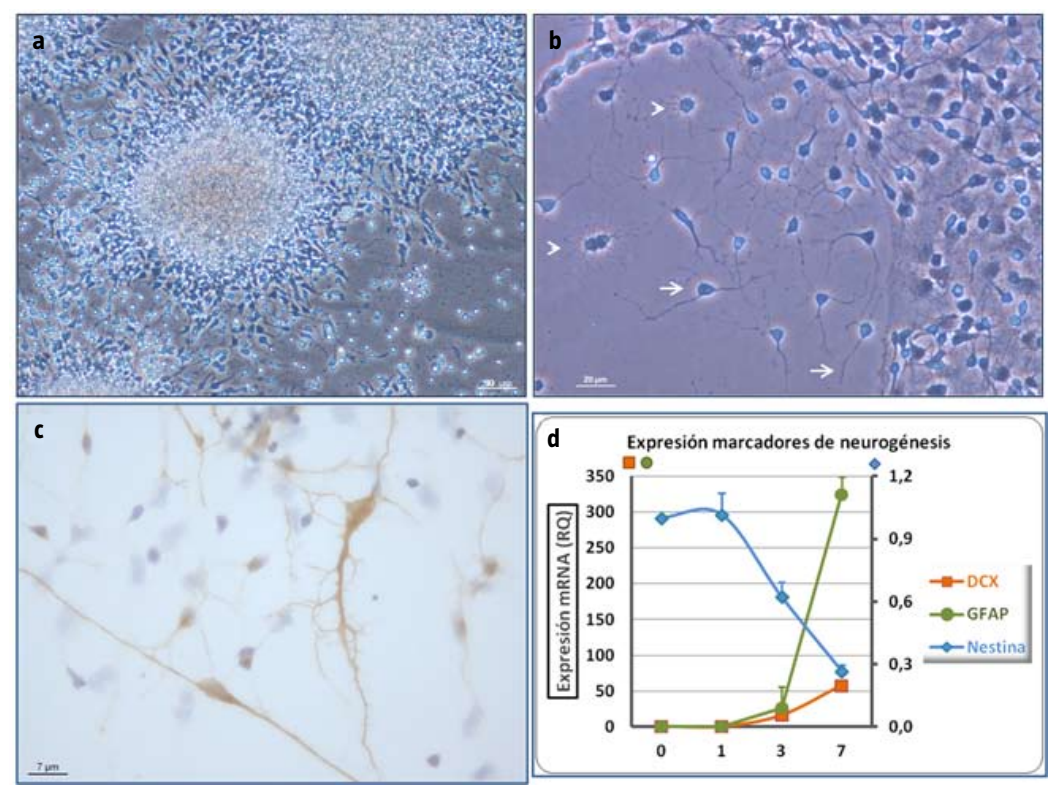

CPN expresan receptores dopaminérgicos $D_{3}$ que responden a la estimulación con apomorfina, produciendo un aumento de la proliferación. Los receptores $\mathrm{D}_{3}$ se coexpresan con nestina y B3T principalmente, y raramente con GFAP, lo que sugiere que el receptor puede estar implicado en la diferenciación a neuronas in vitro. Diversos autores [21,23] indican que el receptor $D_{3}$ se expresa en células precursoras en la ZSV, pero no así en neuroblastos $o$ astrocitos del estriado. Por otro lado, los receptores $\mathrm{D}_{1}, \mathrm{D}_{2}, \mathrm{D}_{4}$ y $\mathrm{D}_{5}$ también se expresan por los neuroblastos. In vivo, el antagonismo farmacológico del receptor $\mathrm{D}_{3}$ disminuye la proliferación en la ZSV y la cantidad de interneuronas que expresan calbindina, calretinina y tiroxina hidroxilasa marcadas con BrdU en el bulbo olfatorio cuatro semanas después del tratamiento. Por otro lado, la ablación, mediante inyección de 6-OHDA o MPTP, de las aferencias dopaminérgicas procedentes de la sustancia negra, resulta en un decrecimiento en la proliferación de la ZSV, lo que sugiere que la actividad de las neuronas de la sustancia negra controla la proliferación celular y la neurogénesis [32-34].
Se piensa que la serotonina (5-HT) tiene un efecto positivo sobre la neurogénesis de la ZSV, ya que su infusión in vitro incrementa la producción de neuroesferas en la ZSV [35]. La activación aguda o crónica de los receptores de serotonina $5-\mathrm{HT}_{1 \mathrm{~A}} \mathrm{y}$ $5-\mathrm{HT}_{2}$, mediante agonistas específicos, incrementa el número de células marcadas con BrdU en la ZSV y el bulbo olfatorio, sin afectar la proporción de glía o nuevas neuronas, mientras que la activación de los receptores $5-\mathrm{HT}_{1 \mathrm{~B}}$ bloquea la proliferación en la ZSV [20]. Sin embargo, la activación crónica de los receptores $5-\mathrm{HT}_{1 \mathrm{~A}}$ puede impedir la migración de las nuevas células hacia el bulbo olfatorio [36].

El adenosín trifosfato (ATP) desempeña un importante papel en el desarrollo del sistema nervioso central a través de la activación de los receptores purinérgicos de dos familias: la $\mathrm{P} 2 \mathrm{X}\left(\mathrm{P} 2 \mathrm{X}_{1-7}\right)$ de receptores ionotrópicos y la P2Y (P2Y $\left.\mathrm{P}_{1-4,6,11-14}\right)$ de receptores metabotrópicos acoplados a proteínas $\mathrm{G}$. En la ZSV, células similares a astrocitos expresan ectonucleotidasas que hidrolizan ATP, lo que sugiere la presencia de señalización purinérgica en la ZSV [37]. A pesar de que existe muy poca información sobre la función de estos receptores en la ZSV, evidencias recientes han demostrado que los cultivos de neuroesferas producen liberación de ATP. También se ha observado recientemente que los neuroblastos adquieren los receptores P2X antes de entrar en la red sináptica del bulbo olfatorio, y que estos receptores podrían mediar una función en la diferenciación de neuroblastos que aún no se ha explorado [38].

\section{Neurogénesis como posible estrategia restauradora en la isquemia cerebral}

\section{Neurogénesis postisquémica: reclutamiento de las} células precursoras neurales a las zonas lesionadas

¿Existe una correcta integración de los circuitos neuronales asociada a la neurogénesis funcional? Como consecuencia de la isquemia, los neuroblastos se desvían de la corriente migratoria rostral hacia el hemisferio lesionado, y diferentes grupos han realizado importantes trabajos en la caracterización de este proceso [39-45]. Arvidsson et al [39] demostraron que, después de una lesión unilateral, las CPN proliferan y migran hacia el estriado lesionado y pueden presentar marcadores de células maduras, como Meis 2 y Pbx, dos semanas después de la recuperación. Zhang et al [46] mostraron, además, que las CPN penetran en el estriado usando un patrón exploratorio en el que una de ellas emite proyeccio- 
nes que presumiblemente le permiten evaluar las condiciones del medio. Posteriormente, es seguida por otras células, formando una cadena similar a lo que ocurre en la corriente migratoria rostral. Además, el proceso de migración se relaciona íntimamente con el de angiogénesis. Thored et al [47] han evidenciado que los neuroblastos se apoyan físicamente en las células endoteliales durante su migración.

Se estima que los cambios en la migración pueden estar influenciados por factores liberados como consecuencia de la isquemia que podrían regular directa o indirectamente este proceso, como el BDNF, VEGF, factor de crecimiento insulínico-1 (IGF-1), TGF $\beta$, factor de necrosis tumoral- $\alpha$ (TNF $\alpha$ ) y factor derivado de células estromales (SDF1 $\alpha$ ), aunque no está claro el mecanismo molecular por el que esto ocurre [48-53]. Estos factores pueden ser segregados por células endoteliales, neuroblastos, astrocitos o microglía, lo que indica que el proceso depende de la interacción entre la totalidad de los tipos celulares implicados en la unidad neurovascular.

El BDNF puede ser liberado por neuronas o astrocitos en respuesta a la isquemia [13,48-52], y tiene claros efectos sobre el daño isquémico. Por ejemplo, mejora la coordinación locomotora, disminuye el número de células apoptóticas en la penumbra, reduce el volumen del infarto y aumenta el número de neuroblastos en el estriado y el borde de la penumbra, efecto acompañado de una reducción del daño neurológico a lo largo de seis semanas [54,55].

El VEGF se incrementa rápidamente después de la isquemia en la corteza [56]. El uso de diferentes estrategias para promover su acumulación después de la isquemia, como la inyección intraventricular en el hemisferio isquémico, la expresión de VEGF recombinante bajo el control del promotor constitutivo del citomegalovirus o la estimulación secundaria de su liberación por administración de eritropoyetina humana recombinante [57] o atorvastatina [58], conlleva un incremento del número de células marcadas con BrdU en la ZSV. De éstas, aquellas células que alcanzaron el límite entre la corteza y el cuerpo calloso isquémicos expresaron los marcadores TUJ-1, MAP2, GAD67 hasta ocho semanas después de la lesión. En paralelo, los tratamientos aumentan la coordinación motora y disminuyen el volumen del infarto.

En un modelo de implantación de neuroesferas sobre cultivos organotípicos que contenían corteza y estriado sometidos a isquemia, Robin et al [48] han puesto de manifiesto que SDF1 $\alpha$ aumenta un $73 \%$ en las rodajas isquémicas comparadas con las no isquémicas, y duplica la distancia recorrida por los neuroblastos a lo largo del tejido lesionado [48].
Curiosamente, la promoción de la migración depende de la cantidad de SDF1 $\alpha$, pues 500 ng de esta quimiocina añadidos al medio triplican el número de neuroblastos en migración, pero su uso combinado con el inhibidor de la proteasa de SDF1 $\alpha$, DPPIV y aprotinina A revierte este efecto, lo que implica un efecto directo del sistema CXCR4/SDF1 en la isquemia cerebral [53]. Por su parte, la inhibición del receptor CXCR4 mediante el uso de anticuerpos bloquea completamente la migración de los neuroblastos [53]. La expresión de SDF1 $\alpha$ y angiopoyetina (Ang-1) aumenta en los vasos sanguíneos tras el ictus, y la acción trópica de estas dos moléculas a través de sus receptores recluta neuronas inmaduras a la corteza del periinfarto. Por tanto, neurogénesis y angiogénesis están causalmente ligadas a través de la producción de SDF1 $\alpha$ y Ang-1. Ambas citocinas promueven la migración de neuroblastos tras la isquemia y la recuperación neurológica [42,59]. Así, el SDF1 $\alpha$ también puede ser liberado desde la glía reactiva en el nicho neurovascular [53,60]. El SDF1 $\alpha$ y la Ang-1 promueven la migración de neuroblastos tras la isquemia y la recuperación del comportamiento, y el SDF1 $\alpha$ puede, además, promover la liberación de glutamato desde los astrocitos en el cuerpo calloso isquémico mediante un proceso exocitótico por la activación del receptor CXCR4 [60].

\section{¿Puede la hipoxia favorecer \\ la neurogénesis en humanos?}

Los efectos de la hipoxia sobre la neurogénesis pueden ser estudiados in vitro, ya que las neuroesferas son resistentes a ella. Chen et al [61] sometieron neuroesferas a diferentes períodos de hipoxia y encontraron que la reoxigenación durante varios días producía un aumento en la viabilidad y proliferación celular. Asimismo, las esferas sufrían un incremento notable del diámetro y un aumento de hasta el 20\% en la incorporación de BrdU después de 12 horas de hipoxia. Curiosamente, sólo las esferas sometidas a 24 horas de hipoxia sufrieron un aumento en el índice de apoptosis, mientras que las esferas sometidas a 6 y 12 horas de hipoxia no la sufrieron. Notablemente, Harms et al [57] determinaron que las neuronas en cultivo son más resistentes a la privación de oxígeno y glucosa si son cocultivadas con neuroesferas también sometidas a ella, efecto mediado por la liberación de VEGF desde estas últimas.

Santilli et al [62], utilizando una línea inmortalizada de CPN humanas, mostraron que la hipoxia leve (2,5-5\% $\left.\mathrm{O}_{2}\right)$ puede favorecer la proliferación de CPN, y la diferenciación de éstas a neuronas y oligodendrocitos frente a astrocitos. Sin embargo, la mayor parte de estas neuronas presenta un fenoti- 
po gabérgico. Curiosamente, las concentraciones de 1 y $20 \%$ de $\mathrm{O}_{2}$ indujeron un aumento significativo en la activación de las caspasas 3 y 9 y en la ruptura de la enzima PARP, pero no se encontraron alteraciones en el potencial de membrana mitocondrial, lo que indica que en la hipoxia grave o en la normoxia las CPN son más susceptibles a morir por apoptosis que en la hipoxia leve. Asimismo, en pacientes de ictus, las células que expresan marcadores asociados con las neuronas nacientes están presentes en la penumbra isquémica alrededor de los infartos corticales, donde estas células se localizan preferentemente en la vecindad de los vasos sanguíneos. Estos hallazgos sugieren la existencia de una neurogénesis compensatoria en el cerebro humano capaz de contribuir a la recuperación postisquémica que representa una diana para la terapia del ictus [63]. En conjunto, estas evidencias sugieren que la hipoxia leve puede favorecer la formación de neuronas a partir de CPN humanas inmortalizadas, abriendo un interesante camino para su uso en terapia regenerativa.

\section{¿Pueden los neurotransmisores promover el proceso de neurogénesis subsiguiente a un ictus?}

Hasta ahora, existen muy pocos trabajos que vinculen la actividad cortical en el hemisferio lesionado directamente con el proceso de neurogénesis. En ratas sometidas a oclusión transitoria de la arteria cerebral media que son entrenadas en cintas de correr durante 14 días después de la isquemia, se observan mayores niveles de glutamato, BDNF y sinapsina 1 en el caudado-putamen, y un mejor desempeño de diversas pruebas motoras que en las ratas no entrenadas [44]. Curiosamente, Centonze et al [64] encontraron un aumento de potenciales excitatorios postsinápticos espontáneos en esta estructura tras inducir oclusión transitoria de la arteria cerebral media. También, la estimulación de los bigotes de ratas produjo un aumento en la concentración de SDF1 $\alpha$ y VEGF, así como un incremento en la migración de neuroblastos a lo largo del cuerpo calloso isquémico y de la incorporación de nuevas neuronas a la corteza isquémica [40]. Por otra parte, Lai et al [65] encontraron que, tras ocho semanas de recuperación, los neuroblastos manifiestan potenciales de acción y son sensibles a la tetrodotoxina. Además, la simple introducción de las ratas en un ambiente enriquecido durante la recuperación induce un ligero incremento en el número de nuevas interneuronas en la corteza lesionada, aunque en este caso la mayor parte de las células generadas fueron de fenotipo glial [66].
Una evidencia más directa de la implicación del glutamato en este proceso la aportaron Di GiorgiGerevini et al [31], al indicar que la depleción del receptor mGluR5, o su inhibición farmacológica mediante MCPG, disminuye el número de neuroblastos presentes en el cuerpo calloso isquémico durante la reperfusión en un modelo de isquemia transitoria. Además, la inhibición in vitro del receptor mGluR5 induce apoptosis en progenitores que están formando neuronas, mientras que la inhibición del receptor mGluR1 bloquea el ciclo celular e induce a la neurodiferenciación [30].

Consideradas conjuntamente, estas evidencias sugieren que la activación corticoestriatal por diferentes tipos de estimulación puede promover la neurorreparación, ya sea por formación de nuevas sinapsis de las neuronas supervivientes debida a la exposición a factores tróficos, o como resultado de un proceso neurogénico que estaría también influenciado por la respuesta combinada a dichos factores tróficos y a la actividad glutamatérgica.

Posible papel de los transportadores vesiculares y de membrana de glutamato en la reestructuración nerviosa y neurogénesis postictus: hipótesis

Es bien conocido el papel del glutamato como desencadenante de la cascada excitotóxica que ocurre durante la isquemia. Recientemente, se ha demostrado que durante las fases iniciales de recuperación, la actividad de los transportadores de membrana plasmática de glutamato (EAAT), es fundamental en la eliminación del exceso de glutamato hacia el plasma, en donde éste es convertido a aspartato por la enzima glutámico oxalacético transaminasa $[67,68]$, proceso que ayuda a reducir el volumen del infarto.

Sin embargo, como hemos mencionado, a largo plazo la actividad glutamatérgica podría ser muy necesaria en la recuperación funcional. Recientemente hemos demostrado que la isquemia focal transitoria en ratas promueve la acumulación de VGLUT1 en la corteza y caudado-putamen, y la expresión de VGLUT2 y 3 en la glía reactiva del cuerpo calloso en el hemisferio isquémico [45]. El incremento observado en la expresión de VGLUT1 mantiene una correlación positiva con la expresión del transportador de membrana EAAT2 y con el daño neurológico (Fig. 4), lo que sugiere que la regulación de la liberación de glutamato a través de VGLUT1 en las fases iniciales de reperfusión podría abordarse como diana terapéutica.

Por otro lado, la presencia de VGLUT2 y 3 en la glía reactiva del cuerpo calloso isquémico durante 
la reperfusión [45] ocurre en paralelo con la expresión de EAAT también en células gliales en la misma estructura [69]. Nosotros hemos encontrado, además, la presencia de ambos sistemas de transporte en cultivos de CPN derivadas de la ZSV, y hemos observado que la expresión de VGLUT (Fig. 5) y EAAT es paralela al incremento en la expresión de DCX, GFAP, NCAM, B3T y disminución de la expresión de nestina (manuscrito en preparación). Además, la activación de receptores NMDA en neuroblastos es fundamental para su supervivencia en la corriente migratoria rostral [27], acción que podría estar mediada por Rho GTPasas, las cuales controlan la correcta diferenciación de las CPN de la ZSV [70] y responden a señales de calcio mediadas por los receptores NMDA [71]. Así pues, nosotros consideramos que la liberación de glutamato en el cuerpo calloso isquémico a través de los VGLUT podría estar vinculada al proceso de neurogénesis en la isquemia.

Por lo tanto, a través de un posible control sobre la actividad de VGLUT1, la regulación de la liberación de glutamato en la corteza en etapas tempranas de reperfusión podría moderar el daño excitotóxico. Por el contrario, durante el período de rehabilitación, la liberación de glutamato en el cuerpo calloso isquémico a través de VGLUT2 y 3, y por VGLUT1 en la corteza, podría influenciar positivamente las diferentes etapas de la neurogénesis hasta llevar a un mayor número de nuevas neuronas integradas sinápticamente.

\section{Conclusiones}

Gran parte del trabajo realizado para desarrollar medicamentos que protejan al cerebro de la isquemia se ha orientado a inhibir la actividad glutamatérgica o a la interferencia de la cascada de señales intracelulares que activan la apoptosis, aunque sin éxito [72]. Por ello, actualmente se está desarrollando la investigación orientada hacia la neurorreparación [1]. Aproximaciones destacables son, por ejemplo, el reclutamiento endógeno de células progenitoras derivadas de la médula ósea [1] o su implantación sobre la zona lesionada a partir de células madre obtenidas de explantes estriatales de fetos humanos o por transdiferenciación de células de otros tejidos [73,74], o bien a través de la potenciación de la migración de neuroblastos desde la ZSV hacia la zona lesionada $[42,52,75]$. Este complejo proceso depende de la interacción de diversos tipos celulares (unión neurovascular) mediante la señalización a través de gran variedad de factores tróficos
Figura 4. Correlación entre los cambios en la expresión de VGLUT1 (1-3d) y EAAT2 (1d) con el deterioro neurológico $(a, b)$ y entre sí $(c)$ en el caudado-putamen de ratas isquémicas.
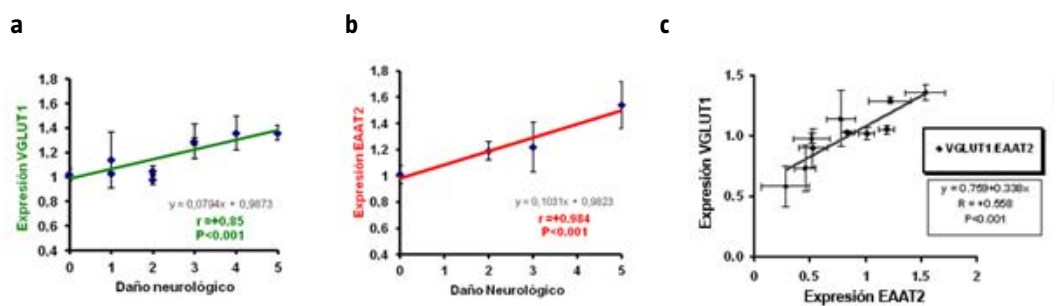

Figura 5. Presencia de VGLUT1-3 en células precursoras neurales. Doble inmunomarcaje de doblecortina (rojo) con VGLUT1-3 (verde) y DAPI (azul) a tres días de diferenciación. Barra de escala: $10 \mu \mathrm{m}$.

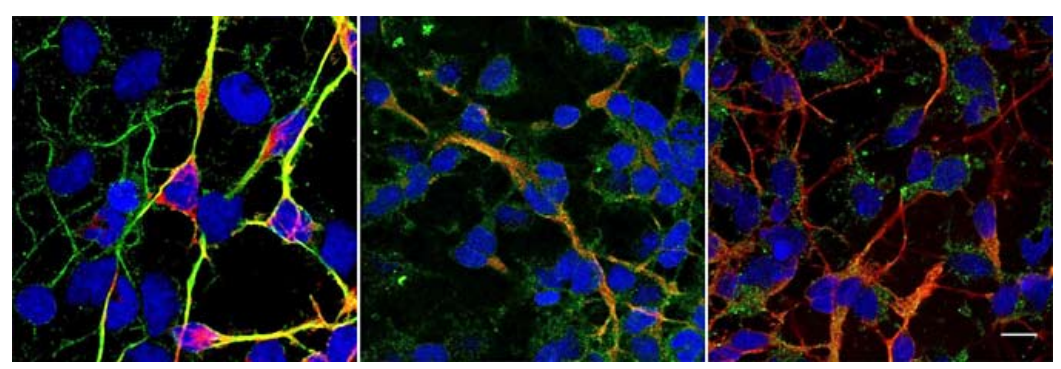

que atraen a las CPN hacia el hemisferio afectado. En el contexto de la neurorreparación, la principal dificultad hoy en día no es sólo incrementar la proliferación y supervivencia de las CPN, sino garantizar su adecuada diferenciación a neuroblastos y neuronas e integración sináptica, evitando, al mismo tiempo, la formación de tumores. En este sentido, diversas evidencias directas e indirectas sugieren la participación de distintos neurotransmisores, y en particular del glutamato, como posibles actores en este proceso, si bien aún no conocemos con exactitud si su actividad le implicaría más en la supervivencia, migración o diferenciación e integración sináptica de los neuroblastos. Así pues, consideramos que una completa caracterización del papel de los neurotransmisores debe ser considerada como una aproximación en la búsqueda de nuevas estrategias de neurorreparación postisquémica.

\section{Bibliografía}

1. Castillo J, Álvarez-Sabín J, Dávalos A, Díez-Tejedor E, Lizasoain I, Martínez-Vila E, et al. Consensus review. Pharmacological neuroprotection in cerebral ischemia: is it still a therapeutic option? Neurologia 2003; 18: 368-84. 
2. Duncan PW, Goldstein LB, Matchar D, Divine GW, Feussner J. Measurement of motor recovery after stroke: outcome assessment and sample size requirements. Stroke 1992; 23: 1084-89.

3. Calautti C, Baron JC. Functional neuroimaging studies of motor recovery after stroke in adults: a review. Stroke 2003, 34: 1553-66.

4. Lichtenwalner RJ, Parent JM. Adult neurogenesis and the ischemic forebrain. J Cereb Blood Flow Metab 2006; 26: 1-20.

5. Agasse F, Bernardino L, Malva J. ZSV cells as a tool for brain repair. In Malva JO, Rego AC, Cunha RA, Oliveira CR, eds. Interaction between neurons and glia in aging and disease. New York: Springer; 2007. p. 81-108.

6. Quiñones-Hinojosa A, Sanai N, Soriano-Navarro M, González-Pérez O, Mirzadeh Z, Gil-Perotin S. Cellular composition and cytoarchitecture of the adult human subventricular zone: a niche of neural stem cells. J Comp Neurol 2006; 494: 415-34.

7. García-Verdugo JM, Doetsch F, Wichterle H, Lim DA Álvarez-Buylla A. Architecture and cell types of the adult subventricular zone: in search of the stem cells. J Neurobiol 1998; 36: 234-48.

8. Arias-Carrión O, Olivares-Buñuelos T, Drucker-Colín R. Neurogénesis en el cerebro adulto. Rev Neurol 2007; 44: 541-50.

9. Abrous DN, Koehl M, Le Moal M. Adult neurogenesis: from precursors to network and physiology. Physiol Rev 2005; 85: 523-69.

10. Sánchez-Mendoza E, Díaz-Castroverde S, Roncero C, Arce C, González MP, Oset-Gasque MJ. Alteraciones en la expresión de los transportadores de glutamato en el proceso de neurogénesis de adulto de la zona subventricular: implicaciones en neurorreparación en la isquemia cerebral [abstract P150]. XIV Congreso Nacional Sociedad Española de Neurociencia. Salamanca, 28-30 de septiembre de 2011.

11. Bagley JA, Belluscio L. Dynamic imaging reveals that brainderived neurotrophic factor can independently regulate motility and direction of neuroblasts within the rostral migratory stream. Neuroscience 2010; 169: 1449-61.

12. Bath KG, Lee FS. Neurotrophic factor control of adult ZSV neurogenesis. Dev Neurobiol 2010; 70: 339-49.

13. Jean YY, Lercher LD, Dreyfus CF. Glutamate elicits release of BDNF from basal forebrain astrocytes in a process dependent on metabotropic receptors and the PLC pathway. Neuron Glia Biol 2008; 4: 35-42.

14. Kaneko N, Marin O, Koike M, Hirota Y, Uchiyama Y, Wu JY, et al. New neurons clear the path of astrocytic processes for their rapid migration in the adult brain. Neuron 2010; 67: 213-23

15. Pathania M, Yan LD, Bordey A. A symphony of signals conducts early and late stages of adult neurogenesis. Neuropharmacology 2010; 58: 865-76.

16. Jeong Y, Mangelsdorf DJ. Nuclear receptor regulation of stemness and stem cell differentiation. Exp Mol Med 2009; 41: 525-37.

17. Sun G, Yu RT, Evans RM, Shi Y. Orphan nuclear receptor TLX recruits histone deacetylases to repress transcription and regulate neural stem cell proliferation. Proc Natl Acad Sci U S A 2007; 104: 15282-7.

18. Qu Q, Sun G, Li W, Yang S, Ye P, Zhao C, et al. Orphan nuclear receptor TLX activates Wnt/beta-catenin signalling to stimulate neural stem cell proliferation and self-renewal. Nat Cell Biol 2010; 12: 31-40.

19. Qu Q, Shi Y. Neural stem cells in the developing and adult brains. J Cell Physiol 2009; 221: 5-9.

20. Banasr M, Hery M, Printemps R, Daszuta A. Serotonininduced increases in adult cell proliferation and neurogenesis are mediated through different and common 5-HT receptor subtypes in the dentate gyrus and the subventricular zone. Neuropsychopharmacology 2004; 29: 450-60.

21. Coronas V, Bantubungi K, Fombonne J, Krantic S, Schiffmann SN, Roger M. Dopamine D3 receptor stimulation promotes the proliferation of cells derived from the post-natal subventricular zone. J Neurochem 2004; 91: 1292-301.

22. Gu W, Gu C, Jiang W, Wester P. Neurotransmitter synthesis in poststroke cortical neurogenesis in adult rats. Stem Cell Res 2009; 4: 148-54.

23. Kim Y, Wang WZ, Comte I, Pastrana E, Tran PB, Brown J, et al. Dopamine stimulation of postnatal murine subventricular zone neurogenesis via the D3 receptor. J Neurochem 2010; 114: 750-60

24. Platel JC, Stamboulian S, Nguyen I, Bordey A. Neurotransmitter signaling in postnatal neurogenesis: the first leg. Brain Res Rev 2010; 63: 60-71.

25. Nakamichi N, TakaradaT, Yoneda Y. Neurogenesis mediated by gamma-amino-butyric acid and glutamate signaling. J Pharmacol Sci 2009; 110: 133-49.

26. Platel JC, Lacar B, Bordey A. GABA and glutamate signaling: homeostatic control of adult forebrain neurogenesis. J Mol Histol 2007; 38: 602-10.

27. Platel JC, Dave KA, Bordey A. Control of neuroblast production and migration by converging GABA and glutamate signals in the postnatal forebrain. J Physiol 2008; 586: 3739-43.

28. Platel JC, Heintz T, Young S, Gordon V, Bordey A. Tonic activation of GLUK5 kainate receptors decreases neuroblast migration in whole-mounts of the subventricular zone. J Physiol 2008; 586: 3783-93.

29. Platel JC, Dave KA, Gordon V, Lacar B, Rubio ME, Bordey A. NMDA receptors activated by subventricular zone astrocytic glutamate are critical for neuroblast survival prior to entering a synaptic network. Neuron 2010; 65: 859-72.

30. Castiglione M, Calafiore M, Costa L, Sortino MA, Nicoletti F, Copani A. Group metabotropic glutamate receptors control proliferation, survival and differentiation of cultured neural progenitor cells isolated from the subventricular zone of adult mice. Neuropharmacology 2008; 55: 560-7.

31. Di Giorgi-Gerevini V, Melchiorri D, Battaglia G, Ricci-Vitiani L, Ciceroni C, Busceti CL, et al. Endogenous activation of metabotropic glutamate receptors supports the proliferation and survival of neural progenitor cells. Cell Death Differ 2005 12: 1124-33.

32. Baker SA, Baker KA, Hagg T. Dopaminergic nigrostriatal projections regulate neural precursor proliferation in the adult mouse subventricular zone. Eur J Neurosci 2004; 20: 575-9.

33. Cova L, Armentero MT, Zennaro E, Calzarossa C, Bossolasco P, Busca G, et al. Multiple neurogenic and neurorescue effects of human mesenchymal stem cell after transplantation in an experimental model of Parkinson's disease. Brain Res 2010; 1311: 12-27.

34. O'Keeffe GC, Barker RA, Caldwell MA. Dopaminergic modulation of neurogenesis in the subventricular zone of the adult brain. Cell Cycle 2009; 8: 2888-94.

35. Hitoshi S, Maruta N, Higashi M, Kumar A, Kato N, Ikenaka K. Antidepressant drugs reverse the loss of adult neural stem cells following chronic stress. J Neurosci Res 2007; 85: 3574-85.

36. Soumier A, Banasr M, Goff LK, Daszuta A. Region- and phase-dependent effects of 5-HT(1A) and 5-HT(2C) receptor activation on adult neurogenesis. Eur Neuropsychopharmaco 2010; 20: 336-45.

37. Lin JH, Takano T, Arcuino G, Wang X, Hu F, Darzynkiewicz Z, et al. Purinergic signaling regulates neural progenitor cell expansion and neurogenesis. Dev Biol 2007; 302: 356-66.

38. Young SZ, Taylor MM, Bordey A. Neurotransmitters couple brain activity to subventricular zone neurogenesis. Eur J Neurosci 2011; 33: 1123-32.

39. Arvidsson A, Collin T, Kirik D, Kokaia Z, Lindvall O. Neuronal replacement from endogenous precursors in the adult brain after stroke. Nat Med 2002; 8: 963-70.

40. Agasse F, Roger M, Coronas V. Neurogenic and intact or apoptotic non-neurogenic areas of adult brain release diffusible molecules that differentially modulate the development of subventricular zone cell cultures. Eur J Neurosci 2004; 19: 1459-68.

41. Agasse F, Bernardino L, Kristiansen H, Christiansen SH, Ferreira R, Silva B, et al. Neuropeptide Y promotes neurogenesis in murine subventricular zone. Stem Cells 2008; 26: 1636-45.

42. Li WL, Yu SP, Ogle ME, Ding XS, Wei L. Enhanced neurogenesis and cell migration following focal ischemia 
and peripheral stimulation in mice. Dev Neurobiol 2008; 68: 1474-86.

43. Zhang RL, Zhang ZG, Chopp M. Ischemic stroke and neurogenesis in the subventricular zone. Neuropharmacology 2008; 55: 345-52.

44. Chang HC, Yang YR, Wang SG, Wang RY. Effects of treadmill training on motor performance and extracellular glutamate level in striatum in rats with or without transient middle cerebral artery occlusion. Behav Brain Res 2009; 205: 450-5.

45. Sánchez-Mendoza E, Burguete MC, Castelló-Ruiz M, González MP, Roncero C, Salom JB, et al. Transient focal cerebral ischemia significantly alters not only EAATs but also VGLUTs expression in rats: relevance of changes in reactive astroglia. J Neurochem 2010; 113: 1343-55.

46. Zhang RL, Chopp M, Gregg SR, Toh Y, Roberts C, Letourneau Y, et al. Patterns and dynamics of subventricular zone neuroblast migration in the ischemic striatum of the adult mouse. J Cereb Blood Flow Metab 2009; 29: 1240-50.

47. Thored P, Wood J, Arvidsson A, Cammenga J, Kokaia Z, Lindvall O. Long-term neuroblast migration along blood vessels in an area with transient angiogenesis and increased vascularisation after stroke. Stroke 2007; 38: 3032-9.

48. Robin AM, Zhang ZG, Wang L, Zhang RL, Katakowski M, Zhang L, et al. Stromal cell-derived factor 1alpha mediates neural progenitor cell motility after focal cerebral ischemia. J Cereb Blood Flow Metab 2006; 26: 125-34.

49. Bernardino L, Agasse F, Silva B, Ferreira R, Grade S, Malva JO. Tumor necrosis factor-alpha modulates survival, proliferation, and neuronal differentiation in neonatal subventricular zone cell cultures. Stem Cells 2008; 26: 2361-71.

50. Leker RR, Lasri V, Chernoguz D. Growth factors improve neurogenesis and outcome after focal cerebral ischemia. J Neural Transm 2009; 116: 1397-402.

51. Madri JA. Modeling the neurovascular niche: implications for recovery from CNS injury. J Physiol Pharmacol 2009; 60 (Suppl 4): S95-104.

52. Harms KM, Li L, Cunningham LA. Murine neural stem/ progenitor cells protect neurons against ischemia by HIF$1 \alpha$-regulated VEGF signaling. PLoS One 2010; 5: e9767.

53. Merino JJ, Gutiérrez-Fernández M, Rodríguez-Frutos B Álvarez-Grech J, Alcalde ME, Vallejo-Cremades MT, et al. CXCR4/SDF-1 $\alpha$-chemokine regulates neurogenesis and/or angiogenesis within the vascular niche of ischemic rats; however, does SDF-1 $\alpha$ play a role in repair? Int J Stroke 2011; 6: 466-7.

54. Schabitz WR, Berger C, Kollmar R, Seitz M, Tanay E, Kiessling M, et al. Effect of brain-derived neurotrophic factor treatment and forced arm use on functional motor recovery after small cortical ischemia. Stroke 2004; 35: 992-7.

55. Saarelainen T, Lukkarinen JA, Koponen S, Grohn OH, Jolkkonen J, Koponen E, et al. Transgenic mice overexpressing truncated trkB neurotrophin receptors in neurons show increased susceptibility to cortical injury after focal cerebral ischemia. Mol Cell Neurosci 2000; 16: 87-96.

56. Hayashi T, Abe K, Suzuki H, Itoyama Y. Rapid induction of vascular endothelial growth factor gene expression after transient middle cerebral artery occlusion in rats. Stroke 1997; 28: 2039-44

57. Wang L, Zhang Z, Wang Y, Zhang R, Chopp M. Treatment of stroke with erythropoietin enhances neurogenesis and angiogenesis and improves neurological function in rats. Stroke 2004; 35: 1732-37.

58. Chen J, Zhang C, Jiang H, Li Y, Zhang L, Robin A, et al. Atorvastatin induction of VEGF and BDNF promotes brain plasticity after stroke in mice. J Cereb Blood Flow Metab 2005; 25: 281-90.
59. Ohab JJ, Fleming S, Blesch A, Carmichael ST. A neurovascular niche for neurogenesis after stroke. J Neurosci 2006; 26: 13007-16.

60. Cali C, Marchaland J, Regazzi R, Bezzi P. SDF 1-alpha (CXCL12) triggers glutamate exocytosis from astrocytes on a millisecond time scale: imaging analysis at the single-vesicle level with TIRF microscopy. J Neuroimmunol 2008; 198: 82-91.

61. Chen X, Tian Y, Yao L, Zhang J, Liu Y. Hypoxia stimulates proliferation of rat neural stem cells with influence on the expression of cyclin D1 and c-Jun N-terminal protein kinase signaling pathway in vitro. Neuroscience 2010; 165: 705-14.

62. Santilli G, Lamorte G, Carlessi L, Ferrari D, Rota Nodari L, Binda E, et al. Mild hypoxia enhances proliferation and multipotency of human neural stem cells. PLoS One 2010; 5: e8575.

63. Jin K, Wang X, Xie L, Mao XO, Zhu W, Wang Y, et al. Evidence for stroke-induced neurogenesis in the human brain. Proc Natl Acad Sci U S A 2006; 103: 13198-202.

64. Centonze D, Rossi S, Tortiglione A, Picconi B, Prosperetti C, De Chiara V, et al. Synaptic plasticity during recovery from permanent occlusion of the middle cerebral artery. Neurobiol Dis 2007; 27: 44-53.

65. Lai B, Mao XO, Xie L, Jin K, Greenberg DA. Electrophysiological neurodifferentiation of subventricular zone-derived precursor cells following stroke. Neurosci Lett 2008; 442: 305-8.

66. Komitova M, Perfilieva E, Mattsson B, Eriksson PS, Johansson BB. Enriched environment after focal cortical ischemia enhances the generation of astroglia and NG2 positive polydendrocytes in adult rat neocortex. Exp Neurol 2006; 199: 113-21.

67. Campos F, Sobrino T, Ramos-Cabrer P, Castellanos M, Blanco M, Rodríguez-Yáñez M, et al. High blood glutamate oxaloacetate transaminase levels are associated with good functional outcome in acute ischemic stroke. J Cereb Blood Flow Metab 2011; 31: 1387-93.

68. Campos F, Sobrino T, Ramos-Cabrer P, Castillo J. Oxaloacetate: a novel neuroprotective for acute ischemic stroke. Int J Biochem Cell Biol 2012; 44: 262-5.

69. Arranz AM, Gottlieb M, Pérez-Cerdá F, Matute C. Increased expression of glutamate transporters in subcortical white matter after transient focal cerebral ischemia. Neurobiol Dis 2010; 37: 156-65.

70. Leone DP, Srinivasan K, Brakebusch C, McConnell SK. The rho GTPase Rac1 is required for proliferation and survival of progenitors in the developing forebrain. Dev Neurobiol 2010; 70: 659-78.

71. Ponimaskin E, Voyno-Yasenetskaya T, Richter DW, Schachner M, Dityatev A. Morphogenic signaling in neurons via neurotransmitter receptors and small GTPases. Mol Neurobiol 2007; 35: 278-87.

72. Fernández-Gómez FJ, Hernández F, Argandoña L, Galindo MF, Segura T, Jordán J. Farmacología de la neuroprotección en el ictus isquémico agudo. Rev Neurol 2008; 47: 253-60.

73. Darsalia V, Allison SI, Cusulin C, Monni E, Kuzdas D, Kallur T, et al. Cell number and timing of transplantation determine survival of human neural stem cell grafts in stroke-damaged rat brain. J Cereb Blood Flow Metab 2011; 31: 235-42.

74. Ishibashi S, Sakaguchi M, Kuroiwa T, Yamasaki M, Kanemura Y, Shizuko I, et al. Human neural stem/progenitor cells, expanded in long-term neurosphere culture, promote functional recovery after focal ischemia in Mongolian gerbils. J Neurosci Res 2004; 78: 215-23.

75. Wang YQ, Cui HR, Yang SZ, Sun HP, Qiu MH, Feng XY, et al. VEGF enhance cortical newborn neurons and their neurite development in adult rat brain after cerebral ischemia. Neurochem Int 2009; 55: 629-36. 


\section{Brain repair after ischemic stroke: role of neurotransmitters in post-ischemic neurogenesis}

Introduction. Brain ischemia and reperfusion produce alterations in the microenvironment of the parenchyma, including ATP depletion, ionic homeostasis alterations, inflammation, release of multiple cytokines and abnormal release of neurotransmitters. As a consequence, the induction of proliferation and migration of neural stem cells towards the peri-infarct region occurs.

Development. The success of new neurorestorative treatments for damaged brain implies the need to know, with greater accuracy, the mechanisms in charge of regulating adult neurogenesis, both under physiological and pathological conditions. Recent evidence demonstrates that many neurotransmitters, glutamate in particular, control the subventricular zone, thus being part of the complex signalling network that influences the production of new neurons.

Conclusion. Neurotransmitters provide a link between brain activity and subventricular zone neurogenesis. Therefore, a deeper knowledge of the role of neurotransmitters systems, such as glutamate and its transporters, in adult neurogenesis, may provide a valuable tool to be used as a neurorestorative therapy in this pathology.

Key words. Brain ischemia. Glutamate. Neural progenitor cells. Neurogenesis. Neurorepair. Neurotransmitters. 